\title{
DEVELOPMENT FOR LARGE DYNAMICAL FIELD RANGE,WIDE GOOD FIELD REGION C-MAGNET
}

\author{
T. Zhang, China Institute of Atomic Energy, Beijing 102413, China
}

K. Sato, S. Ninomiya, Research Center for Nuclear Physics, Ibaraki, Osaka 567-0047, Japan

H. Saito, Y. Sasaki, S. Morioka, Sumitomo Heavy Industries, Niihama, Ehime 792-8588, Japan

\begin{abstract}
The dipole magnets are used frequently in the different types of accelerators. The solution of Laplace's equation was introduced to calculate the pole shape based on the assumption that the pole surface is the equal scalar potential surface if the permeability is high enough [1]. During the calculation of the pole shape, one usually uses the error function to describe the distribution of the magnetic field. However, we suffered some convergence problem in the solving process of the equal potential equation with the high order derivatives of the error function. In this paper, a new function is opted to describe the distribution of the field for the dipole. The solutions of the 3D Laplace's equation determined by the distribution from this new function are investigated widely. A dipole magnet with the resulting shape of the pole is designed by the numerical computation to confirm the performance of this new function and is fabricated based on such a design for the experiment study. The 2D and integrated field uniformity and the good field region are measured by Hall probe and search coils respectively. The measurement results are also presented in this paper.
\end{abstract}

\section{INTRODUCTION}

The dipole magnets are playing an essential role in many accelerators and their transport lines. If the various species of ions are expected to be accelerated, stored and transported, e.g. the synchrotron for proton, light-heavy ions, polarized ions and electrons, the beam lines for RIB facilities, etc, the dipoles used for these machines are provided with large dynamical field range. It is important to obtain the wide good field region through the whole dynamical field range. The field quality of the dipoles is chiefly based on the pole shape. In RCNP, an effort to improve the field quality is made and a C-magnet is developed, which is based on the pole shape defined by the solutions of 3D Laplace's equation.

\section{C-MAGNET DEVELOPMENT}

\subsection{The solution of Laplace's equation}

The solution of Laplace's equation was introduced to calculate the pole shape. In the current free region, the flux density $\vec{B}$ can be expressed with the scalar potential $\varphi$, which is governed by the Laplace's equation. From the Laplace's equation, the equation used to define the pole surface can be derived if the permeability of the magnet is high enough. During the derivation for the equation of the pole surface, one uses the error function to represent the field distribution and know the expression for the equation of the pole surface written as follows.

$$
\frac{d}{2}=\sum_{\mu=0}^{\infty} \frac{(-1)^{\mu}}{(2 \mu+1) !} y^{2 \mu+1}\left\{\sum_{i=0}^{\mu} C_{i}^{\mu}\left[{\frac{\partial^{2(\mu-i)}}{\partial x}}^{g(x)}\right] \cdot\left[{\frac{\partial^{2 i}}{\partial s}}^{2 i}(s)\right]\right\}
$$

where $d$ is the height of gap, $C_{i}^{\mu}, g(x)$ and $G(s)$ are given by:

$$
\begin{aligned}
C_{i}^{\mu} & =\frac{\mu !}{i !(\mu-i) !} \\
g(x) & =\left[e f r\left(\frac{x+x 0}{\sigma_{x}}\right)-e f r\left(\frac{x-x 0}{\sigma_{x}}\right)\right] / e f r\left(\frac{x 0}{\sigma_{x}}\right) \\
G(s) & =\left[e f r\left(\frac{s+s 0}{\sigma_{s}}\right)-e f r\left(\frac{s-s 0}{\sigma_{s}}\right)\right] / e f r\left(\frac{s 0}{\sigma_{s}}\right)
\end{aligned}
$$

$g(x)$ and $G(s)$ are the field distribution on the median plane along the $x$ and $s$ direction respectively. The coordinates system used in this paper is defined as: $s$, the distance along the reference orbit, $y$, perpendicular to the pole surface, $x$, on the medium plane and perpendicular to $s$.

We observe that the super high order derivatives of the error function should be calculated for solving the Eq. (1). And the derivatives of $g(x)$ and $G(s)$ are coupled together . Such a coupling of derivatives causes some convergence problems. One can truncate $\mu$ at a specific value, but the solution is not reasonable some time if $\mu$ is not high enough. To improve such a equal potential method for the pole shape representation, we introduce a new function to describe the field distribution in the no current source region. They are defined as:

$$
\begin{aligned}
& g(x)=q(x)-f(x) \\
& G(s)=Q(s)-F(s) \\
& q(x)=\frac{1}{2\left(1-\mathrm{e}^{-\frac{x}{\sigma_{x}}}\right)}\left[\frac{x+x 0}{|x+x 0|}-\frac{x-x 0}{|x-x 0|}\right] \\
& f(x)=\frac{1}{2\left(1-\mathrm{e}^{-\frac{\mathrm{x} 0}{\sigma_{\mathrm{x}}}}\right)}\left[\frac{x+x 0}{|x+x 0|} \cdot \mathrm{e}^{-\frac{|x+x 0|}{\sigma_{x}}}-\frac{x-x 0}{|x-x 0|} \cdot \mathrm{e}^{-\frac{|x-x 0|}{\sigma_{x}}}\right]
\end{aligned}
$$




$$
\begin{aligned}
& Q(s)=\frac{1}{2\left(1-\mathrm{e}^{-\frac{s 0}{\sigma_{s}}}\right)}\left[\frac{s+s 0}{|s+s 0|}-\frac{s-s 0}{|s-s 0|}\right], \\
& F(s)=\frac{1}{2\left(1-\mathrm{e}^{-\frac{s 0}{\sigma_{\mathrm{s}}}}\right)}\left[\frac{s+s 0}{|s+s 0|} \cdot \mathrm{e}^{-\frac{|s+s 0|}{\sigma_{\mathrm{s}}}}-\frac{s-s 0}{|s-s 0|} \cdot \mathrm{e}^{-\frac{|s-s 0|}{\sigma_{\mathrm{s}}}}\right]
\end{aligned}
$$

Then, from 3D Laplace's equation, we get:

$$
\begin{aligned}
& \frac{d}{2}=y \cdot g(x) \cdot G(s)+ \\
& \sum_{\mu=1}^{\infty} \frac{(-1)^{\mu}}{(2 \mu+1) !} y^{2 \mu+1}\left\{\sum_{i=0}^{\mu} C_{i}^{\mu}\left[\frac{f(x)}{\sigma_{x}^{2(\mu-i)}}\right] \cdot\left[\frac{F(s)}{\sigma_{s}^{2 i}}\right]\right\}
\end{aligned}
$$

It can be found that the derivatives are disappeared in the Eq. (2). So, we can get the solution with very high $\mu$ by the proper numerical method. We used this improved equal potential method to design a prototype C-magnet. A typical field distribution along the beam direction $s$ defined by the new function is shown in figure. 1 . The pole shape will be fixed after the solving of Eq. (2). Figure 2 is extracted from the design stage, which gives the pole shape of the $\mathrm{C}$-magnet with the different truncated $\mu$. It shows us that the higher $\mu$ is necessary in the practical design. Because the field distribution along $x$ direction is affected strongly by the coils and return yoke, one should not expect to use a single function to represent the field distribution in the whole space. We use two sets of the different parameters to define two distribution functions $g l(x)$ and $g 2(x)$, which represent the field inside and outside the pole respectively.

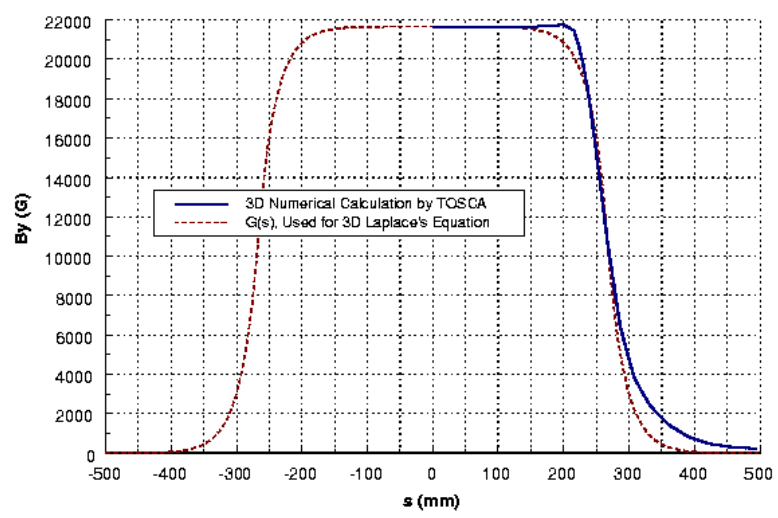

Figure 1: Field distribution along reference orbit.

\subsection{Numerical Design}

Based on the resulting pole shape defined by Eq. (2), a dipole magnet is modeled by TOSCA [2]. The modeled magnet is shown in figure. 3 and the field along $s$ got from TOSCA is also plotted in the figure. 1. The field around $s=200 \mathrm{~mm}$ are different with that from the function $G(s)$. We think it is due to the saturation of the

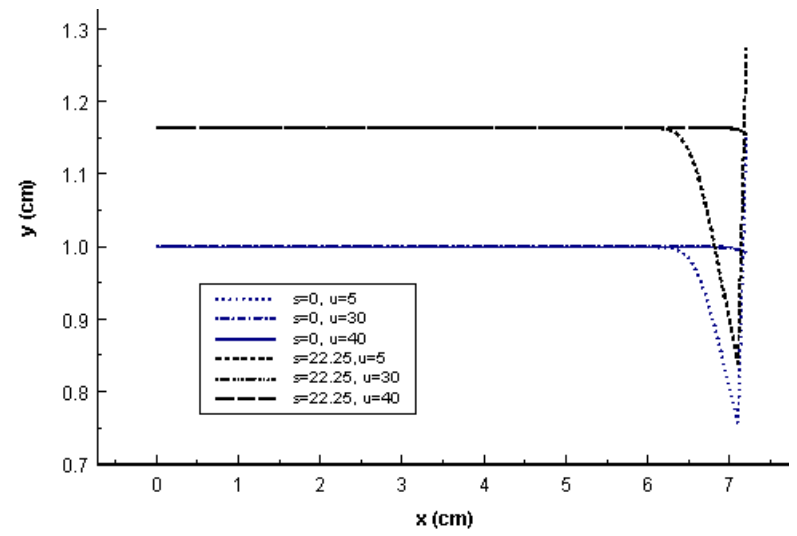

Figure 2: Pole shape calculated by different truncated $\mu$.

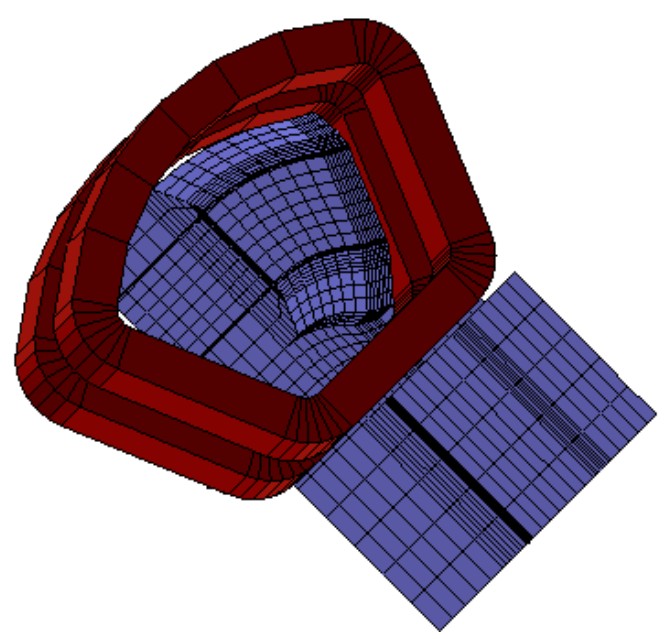

Figure 3: 3D FEM calculation for the C-magnet.

end piece. At $s=350 \mathrm{~mm}$, the higher field are because of the closer coils which also shown in figure 3 . The good field regions for various field intensities are estimated by POISSON [3]. From figure 4, it can be found that the good field regions are improved, especially at the high field range. The pole shapes given by 3D Laplace's equation, used for numerical design and fabrication are compared in figure 5. The numerical results show us that the pole shape, including the end pieces defined by Eq. (2) provides a good field distribution in the gap. The overview parameters of the final design is listed as following:

- $\mathrm{Gap}=2.4 \mathrm{~cm}$

- Width of pole $=17.5 \mathrm{~cm}$

- $\quad$ Angle of bend $=90$ degrees

- Radius of curvature $=34 \mathrm{~cm}$

- Entrance pole rotation angle $=20$ degrees

- Exit pole rotation angle $=20$ degrees

- Field index $n=0$

- Power consumption $=6.1 \mathrm{~kW}$

- Weight $=4.5 \mathrm{t}$

The designed C-magnet is shown in figure 6. 


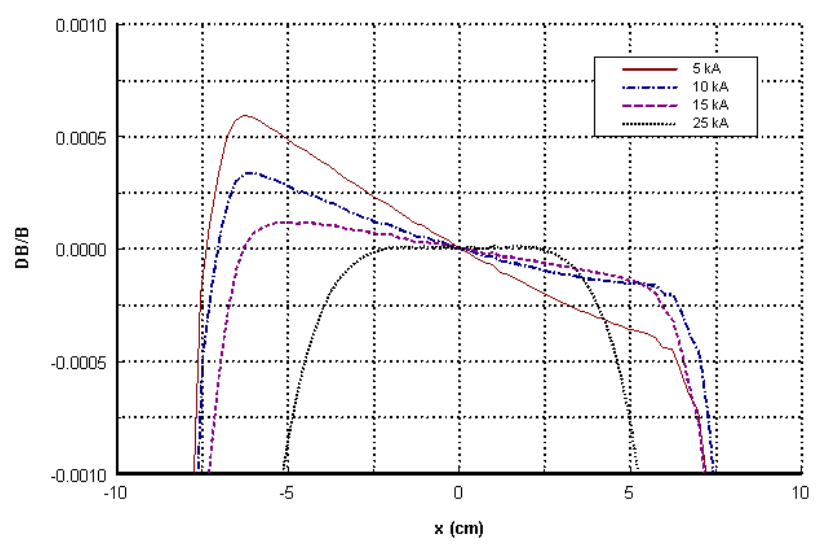

Figure 4: The good field region for various field Intensity.

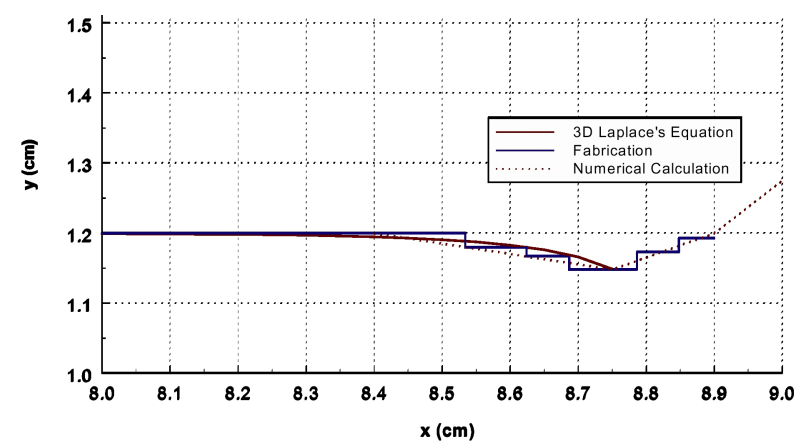

Figure 5: The pole shape, from 3D Laplace's equation to fabrication.

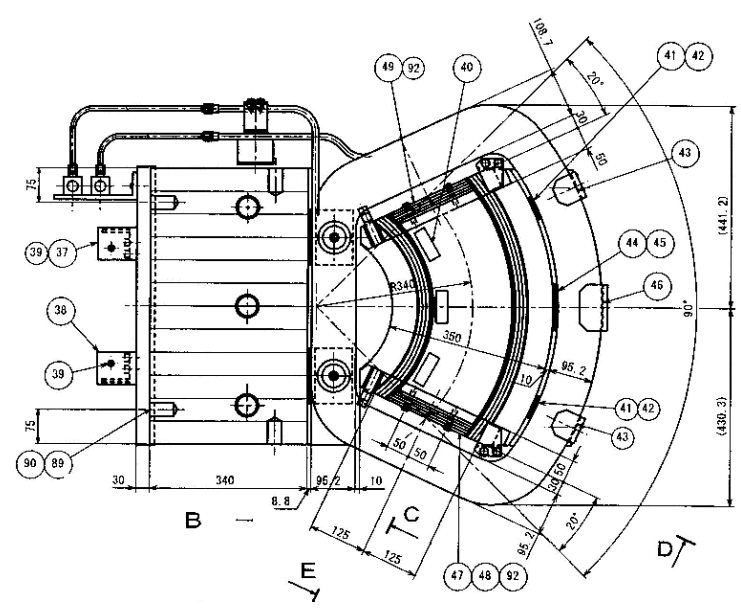

Figure 6: The designed C-magnet

\subsection{Measurement}

The field distribution is measured by the hall probe and search coils. The hall probe is used to measure the B-I characteristic and 2D field uniformity. The integrated field uniformity is measured by a four-search coils system, which is mounted on a moving plate made of the engineering plastic PET 100. The difficulty of the search coil based measurement is that the radius of magnet curvature is so small that we not only need to adjust the position, but also the orientation of the search coils during the measurement. The measurement results of the $2 \mathrm{D}$ and integrated field uniformity with a comparison of the design data are given in table 1 . The uniformity of integrated field are shown in detail in figure 7.

Table 1: The measurement results by Hall probe and search coils

\begin{tabular}{|c|c|c|c|}
\hline Item & Design Goal & 2D field & $\begin{array}{l}\text { integrated } \\
\text { field }\end{array}$ \\
\hline \multirow{2}{*}{$\begin{array}{l}\text { Good Field } \\
\text { Region }\end{array}$} & $\begin{array}{l} \pm 5.0 \mathrm{~cm} \\
(5 \mathrm{kG}-18 \mathrm{kG})\end{array}$ & $\begin{array}{l} \pm 5.0 \mathrm{~cm} \\
(3 \mathrm{kG}-18 \mathrm{kG})\end{array}$ & $\begin{array}{l} \pm 5.0 \mathrm{~cm} \\
(3 \mathrm{kG}-18 \mathrm{kG})\end{array}$ \\
\hline & $\begin{array}{l} \pm 4 . \quad 0 \quad \mathrm{~cm} \\
(>18 \mathrm{kG})\end{array}$ & $\begin{array}{l} \pm 4.3 \mathrm{~cm} \\
(>20 \mathrm{kG})\end{array}$ & $\begin{array}{l} \pm 4.3 \mathrm{~cm} \\
(>20 \mathrm{kG})\end{array}$ \\
\hline Uniformity & $\pm 5 \times 10^{-4}$ & $\pm 5 \times 10^{-4}$ & $\pm 5 \times 10^{-4}$ \\
\hline $\begin{array}{l}\text { Field } \\
\text { Intensity }\end{array}$ & Up to $21 \mathrm{kG}$ & Up to $21 \mathrm{kG}$ & Up to $21 \mathrm{kG}$ \\
\hline
\end{tabular}

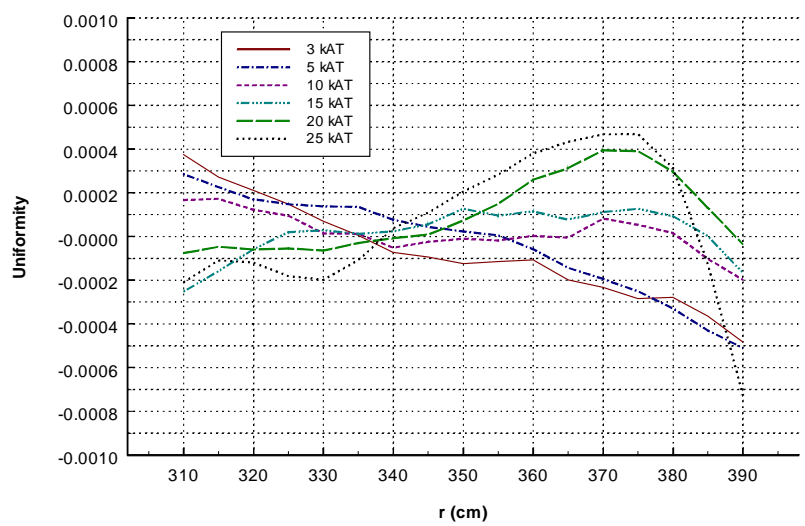

Figure 7: The uniformity of integrated field

\section{SUMMARY}

An improved Laplace's equation method for the dipole magnet design is introduced. Based on it, a C-magnet is designed with the assistance of the numerical method because we ignore the effect of coils and we cannot understand the saturation in the iron by the improved Laplace's equation method. The field distribution of the C-magnet is investigated experimentally. The measurement results have a good agreement with the designed data, which confirms that the pole shape got from the solution of 3D Laplace's equation could provide a wide good field region through the whole dynamical field range.

\section{REFERENCES}

[1] S. Ninomiya, K. Sato, etc., Proc. Of 12th Jap. Symp. on Accelerator Science \& Technology, 1999

[2] OPERA is a finite element code developed by Vector Field Ltd., VF-09-96-D4(1996)

[3] Poisson is a two-dimensional code at the Los Alamos National Laboratory, LA-UR-87-126(1987) 